\title{
Driver-response relationships between frontal EEG and Respiration during affective audiovisual stimuli.
}

\author{
Eleni Kroupi, Jean-Marc Vesin and Touradj Ebrahimi
}

\begin{abstract}
The complementary nature and the coordinative tendencies of brain and body are essential to the way humans function. Although static features from brain and body signals have been shown to reflect emotions, the dynamic interrelation of the two systems during emotional processes is still in its infancy. This study aims at investigating the way brain signals captured by Electroencephalography (EEG) and bodily responses reflected in respiration interact when watching music clips. A non-linear measure is applied to frontal EEG and respiration to determine the driver/driven relationship between these two modalities. The results reveal a unidirectional dependence from respiration to EEG which adds evidence to the bodily-feedback theory.
\end{abstract}

Index Terms-EEG, respiration, affect, non-linear synchronization

\section{INTRODUCTION}

Nature has a ubiquitous tendency to coordinate things. For a living organism to function, its components interact with each other, and self-organize. This should be reflected in the formation of simple and/or complex coupling patterns among various organismic components [1]. For instance, neurons, muscles and joints coordinate in such a way that the entire organism behaves as a coherent unit. This coordination process shifts the attention from the components themselves to the relations among them.

Various aspects of synchronization have been observed in many scientific disciplines, and describe various natural phenomena. Coordination of various components has started to gain attention and interest also in psychology. More specifically, according to appraisal theories of emotion [2], emotional perception of an event occurs when subcomponents of the organism become more synchronized or coupled. For instance, physiological reactions that originate either from the peripheral nervous system (PNS) or from the central nervous system (CNS) are expected to be synchronized during emotional events. PNS reactions usually include respiration, heart rate, body temperature, blood flow and electrodermal activity (EDA), whereas CNS reactions can be assessed using electroencephalography (EEG), magnetoencephalography (MEG), and brain imaging.

*The research leading to these results has been performed in the frameworks of Swiss National Foundation for Scientific Research (FN 200020132673-1), and the NCCR Interactive Multimodal Information Management (IM2).

E. Kroupi and T. Ebrahimi are with the Multimedia Signal Processing Group (MMSPG), École Polytechnique Fédérale de Lausanne (EPFL), EPFL/STI/IEL/GR-EB Station 11, CH-1015 Lausanne.

J. M. Vesin is with the Applied Signal Processing Group (ASPG), École Polytechnique Fédérale de Lausanne (EPFL), EPFL-SCI-STI-JMV, Station 11, CH-1015 Lausanne.
PNS and CNS modalities have been extensively shown to characterize certain emotions. For instance, an increase in heart rate is observed when experiencing emotions such as anger, anxiety, surprise and happiness, while respiration rate as well as skin conductance response seem to increase with anger, anxiety, disgust, fear and amusement [3]. Among peripheral responses, electrodermal activity (EDA) has been extensively used in psychophysiology, due to its particular association with arousal. More specifically, every stimulus accompanied by an emotion produces an alteration of EDA that is proportional to the arousing level of the emotion experienced [4]. Moreover, activity in the ventromedial prefrontal cortex and orbitofrontal cortex covaries with EDA [5], indicating that these brain regions are influenced by sympathetic peripheral arousal. Regarding other brain responses, frontal-lobe asymmetric activity is highly associated with approach/withdrawal emotional processes [6], and acts as a moderator and mediator of emotion [7]. Finally, electroencephalography (EEG) has been widely used to discover associations between emotions and brain activity. In particular, power spectral density in various EEG frequency bands, among other EEG features, has been shown to contain emotional information [8]

Static features from EEG and peripheral signals have been extensively shown to reflect emotional traces [6], [7], [8], [9]. However, the dynamic interrelation of CNS and PNS patterns during emotional processes has received less attention, although there is evidence that brain activation is associated with peripheral and other visceral responses [10]. For instance, several studies [5], [11] have reported frontal lobe activation by EDA, during biofeedback arousing and relaxation tasks. Yet these studies applied linear correlation measures to estimate the interactions between brain activity and EDA, although both EEG and EDA are complex, with non-linear characteristics [12], [13]. Also, a very recent study [14] used phase synchronization to detect the interaction between heart rate and respiration during affective picture viewing. However, both heart rate and respiration emanate from PNS, thus this study provides no information about the interactions between brain activity and bodily responses.

The present work investigates the synchronization between oscillatory brain circuits and peripheral response patterns reflected in EEG signals and respiration, respectively. More specifically, the non-linear interdependence measure presented in [15] is used to capture possible driver/response synchronization patterns between EEG and respiration when experiencing emotional content, in particular, when watching music clips. 
This paper is organized as follows. Section II describes the dataset, the methods used in this study and the proposed approach. The results and the further discussion are detailed in Section III. Finally, the conclusions are presented in Section IV.

\section{MATERIALS AND METHODS}

\section{A. Interdependence algorithm}

The notion of coordination or synchronization can be interpreted in different ways. Typically, synchronization refers to a process wherein two or more dynamical systems adjust some of their properties to a common behavior due to strong or weak coupling. The stricter way of using the concept of synchronization refers to "complete" or "identical" synchronization, and describes the dynamics of two (or more) systems that move along identical orbits [16]. However, this is obviously not the case for systems that are not identical, which leads to the need of adopting looser concepts of synchronization. For instance, "generalized" synchronization may exist among non-identical systems that can be contaminated with noise. Generalized synchronization refers to interdependencies in the state space of the systems, and quantifies how neighboring state vectors of one state space map into the other.

In order to detect the possible generalized synchronization between EEG and respiration, the interdependence measure presented in [15] was used. This measure, unlike other common statistical measures, provides information about the direction of the interdependence [15]. It is formed as follows; let $\mathbf{X}=\left(x_{1}, x_{2}, \ldots, x_{L}\right)$ and $\mathbf{Y}=\left(y_{1}, y_{2}, \ldots, y_{L}\right)$ be two simultaneously observable time series. State vectors of each time series are formed according to the time delay embedding in an $m$-dimensional space [17]. This leads to state vectors $\mathbf{x}_{n}=\left(x_{n}, x_{n-\tau_{1}}, \ldots, x_{n-\left(m_{1}-1\right) \tau_{1}}\right)$ and $\mathbf{y}_{n}=$ $\left(y_{n}, y_{n-\tau_{2}}, \ldots, y_{n-\left(m_{2}-1\right) \tau_{2}}\right)$, where $\tau_{1}$ and $\tau_{2}$ are the time delays, and $m_{1}$ and $m_{2}$ the embedding dimensions for $\mathbf{X}$ and $\mathbf{Y}$, respectively. For each state vector $\mathbf{x}_{n}$, the squared mean Euclidean distance from its $k$ nearest neighbors is defined as

$$
R_{n}^{(k)}(\mathbf{X})=\frac{1}{k} \sum_{j=1}^{k}\left(\mathbf{x}_{n}-\mathbf{x}_{r_{n, j}}\right)^{2},
$$

where $r_{n, j}$ denotes the time indices of the $k$ nearest neighbors of $\mathbf{x}_{n}$. The squared mean conditional Euclidean distance is defined as

$$
R_{n}^{(k)}(\mathbf{X} \mid \mathbf{Y})=\frac{1}{k} \sum_{j=1}^{k}\left(\mathbf{x}_{n}-\mathbf{x}_{s_{n, j}}\right)^{2},
$$

where $s_{n, j}$ denotes the time indices of the $k$ nearest neighbors of $\mathbf{y}_{n}$. The local and global interdependence measures are, then, defined as

$$
S_{n}^{(k)}(\mathbf{X} \mid \mathbf{Y})=\frac{R_{n}^{(k)}(\mathbf{X})}{R_{n}^{(k)}(\mathbf{X} \mid \mathbf{Y})},
$$

and

$$
S^{(k)}(\mathbf{X} \mid \mathbf{Y})=\frac{1}{N} \sum_{n=1}^{N} S_{n}^{(k)}(\mathbf{X} \mid \mathbf{Y}),
$$

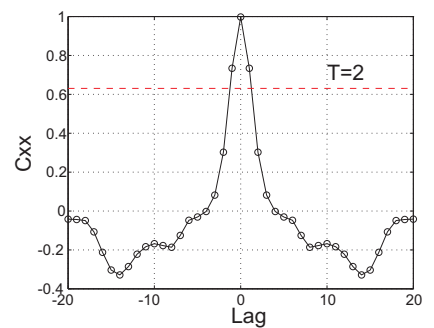

(a)

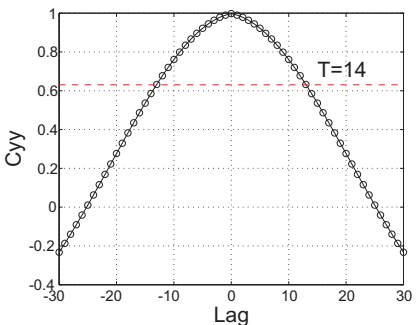

(b)
Fig. 1. Time-lag estimation from the autocorrelation function of an EEG segment (a) and a respiration segment (b).

respectively, where $N$ is the number of state vectors. Since $R_{n}^{(k)}(\mathbf{X} \mid \mathbf{Y}) \geq R_{n}^{(k)}(\mathbf{X})$, then $0<S^{(k)}(\mathbf{X} \mid \mathbf{Y}) \leq 1$. If the dependence of $X$ on $Y$ is high, then $S^{(k)}(\mathbf{X} \mid \mathbf{Y}) \longrightarrow 1$. The opposite dependencies $S_{n}^{(k)}(\mathbf{Y} \mid \mathbf{X})$ and $S^{(k)}(\mathbf{Y} \mid \mathbf{X})$ are defined analogously.

\section{B. Dataset}

The data of 32 participants used in this study was obtained from the DEAP database [18]. The physiological signals were recorded using a Biosemi ActiveTwo system with 32 active $\mathrm{AgCl}$ electrodes, four electrooculogram (EOG) electrodes and a respiration belt, at a sampling frequency of $512 \mathrm{~Hz}$. EEG, EOG and respiration were recorded while forty 1-min music clips (carefully selected) were presented to each participant in a random order, constituting forty single trials.

\section{Signal pre-processing}

EEG, EOG and respiration were downsampled to $128 \mathrm{~Hz}$. EEG and EOG signals were filtered between $3-47 \mathrm{~Hz}$ using a 3rd order Butterworth filter. EOG artifacts were removed using FastICA ${ }^{1}$, and the signals were re-referenced to the common average. Due to the role of the frontal cortex in emotion [7], [6], only the frontal electrodes, namely Fp1, AF3, F3, F7, FC5, FC1, Fp2, AF4, Fz, F4, F8, FC6, and FC2 were used in this study. Furthermore, respiration was filtered between 0.1 and $1 \mathrm{~Hz}$.

\section{Parameter selection and proposed synchronization ap- proach}

The generalized synchronization between EEG and respiration was estimated in the following way; first, to reduce the dimensionality and to retrieve only the most informative part of the frontal EEG signals, principal component analysis (PCA) was applied. Only the PCs that contained $80 \%$ of the signals' variance were used for the analysis. To consider the same range of values, each signal was normalized in the $[-1,1]$ range. Then each PC of each single trial, and the corresponding respiration were segmented using $3-\mathrm{sec}$ overlapping windows (60\% overlap) leading to 49 segments.

To estimate the interdependencies, it was necessary to reconstruct the state vectors for each signal. This was carried

\footnotetext{
${ }^{1}$ http://www.cis.hut.fi/projects/ica/fastica/
} 


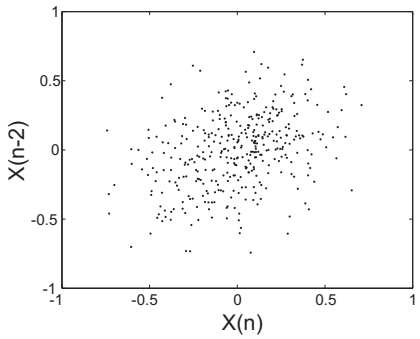

(a)

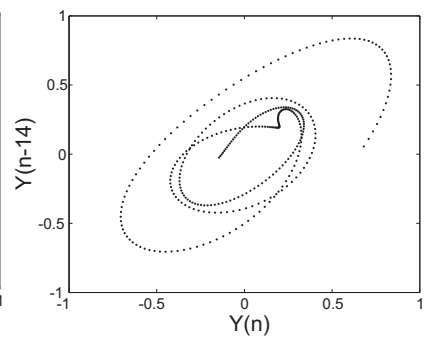

(b)
Fig. 2. An example of EEG state vectors (a), and an example of respiration state vectors (b) embedded in a 2-dimensional space.

out using time-delay embedding [17]. In particular, the timelag for state space reconstruction was estimated using the autocorrelation function of each signal. More specifically, the time-lag was selected as the lag for which the autocorrelation is $1-1 / e$ times its maximum value [17]. An example of the time-lag estimation of an EEG and a respiration segment is presented in Fig. 1.

According to the reconstruction theorem [17], signals have to be embedded in a large Euclidean space $R^{m}$ so that they are unfolded without ambiguity. In the false nearest neighbor (FNN) algorithm [17], one examines increasing embedding dimensions and selects the one for which the number of false neighbors due to improper unfolding decreases drastically. This decision is based on a threshold selected as in the algorithm described in [17]. In particular, according to [17], for a threshold between 10 to 50, the number of false neighbours is constant, indicating that FNN is a stable criterion for selecting the proper embedding dimension since the threshold range is broad enough. In this study the FNN algorithm was applied on each segment, leading to different embedding dimension for each segment. The range of the embedding dimensions for the EEG segments was between 3 to 8 , and between 2 to 3 for the respiration segments. After reconstructing the state vectors of each segment (e.g., Fig. 2(a)-2(b) show the state vectors of an EEG segment and the corresponding respiration segment embedded in a 2-dimensional space), fifty nearest neighbors were used to estimate the interdependencies (eq. (1)-(4)). The final interdependence for each segment was estimated as the average global interdependence between all PCs and the corresponding respiration segments, leading to 49 interdependence values per trial.

\section{RESULTS AND DISCUSSION}

\section{A. Driver/response relationships}

The interdependence measure provides information about the direction of the coupling, due to the fact that it is nonsymmetric [15]. Thus, the goal of this analysis is to investigate whether EEG is the driver or the driven system with respect to respiration during affective audio-visual content. From now on, $S_{x y}$ represents the global interdependence of each single trial, when respiration is the driver and EEG the response. Accordingly, $S_{y x}$ represents the global

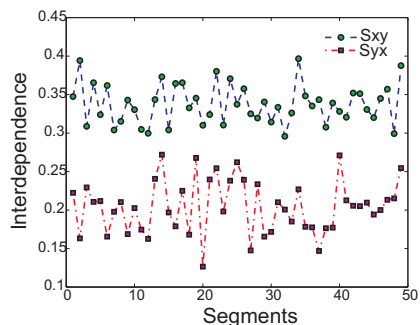

(a)

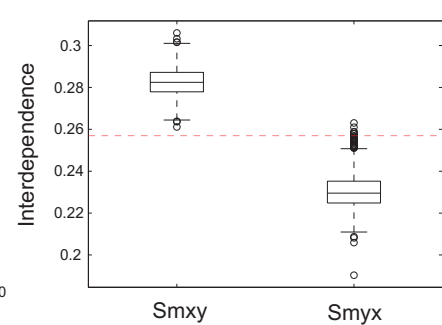

(b)
Fig. 3. (a) Example of how the interdependencies evolve over time both for $S_{x y}$ and for $S_{y x}$. The upper curve corresponds to $S_{x y}$ and the lower one to $S_{y x}$. (b) $S_{m x y}$ and $S_{m y x}$ for all subjects and for $m=3$. The line represents the threshold.

interdependence when EEG is the driver and respiration the response. Each $S_{x y}$ and $S_{y x}$ contains 49 values. Fig. 3(a) displays $S_{x y}$ and $S_{y x}$ for a subject's trial. Differences in the mean and variance of $S_{x y}$ and $S_{y x}$ are visible.

In order to test the significance of the interdependence indexes, the medians of $S_{x y}$ and of $S_{y x}$ were extracted to represent the overall degree of directed synchronization for each single trial. $S_{m x y}$ and $S_{m y x}$ represent the corresponding vectors with the medians of all single trials for each subject, leading to $32 S_{m x y}$ and $S_{m y x}$ vectors with 40 values each. $S_{m x y}$ and $S_{m y x}$ were compared to a proper threshold $t h=$ $(n n / L)^{2 / m}$ [15], where $n n$ is the number of nearest neighbors, $L$ is the length of the segments and $m$ the embedding dimension. $S_{m x y}$ was found to be above and $S_{m y x}$ below the threshold. Following [15], this finding indicates that $S_{m x y}$ describes significantly the interdependencies between EEG and respiration, while $S_{m y x}$ does not. For a fixed $m=3$, the behavior of $S_{m x y}$ and $S_{m y x}$ compared to the corresponding threshold for all subjects is presented in Fig. 3(b).

In addition, a more accurate way to investigate the overall differences between $S_{m x y}$ and $S_{m y x}$ is by applying a statistical analysis. To this regard, a non-parametric bootstrap hypothesis test [19] was applied on each $S_{m x y}$ and $S_{m y x}$. The null hypothesis was that $S_{m x y}$ is lower than $S_{m y x}$. Bonferroni's correction was then applied to the $p$-values, and a Stouffer's weighted meta-analysis test provided the final $p$-value. Stouffer's weighted test combines the $p$-values of independent tests (different subjects) which share the same null hypothesis $\left(S_{m x y}\right.$ is smaller than $\left.S_{m y x}\right)$. This test was selected because it outperforms other meta-analysis tests [20]. The null hypothesis was rejected with $p<0.001$, indicating that, in fact, $S_{m x y}$ is larger than $S_{m y x}$ while watching music clips. Fig. 4 displays $S_{m x y}$ and $S_{m y x}$ for all subjects. Since the existence of interdependent values with significant asymmetry ( $S_{m x y}$ significantly larger than $S_{m y x}$ ) can be interpreted as an indication of unidirectional coupling, we can speculate that EEG depends more on respiration than vice versa during music clips.

\section{B. Discussion}

From a mathematical point of view, if $S_{m x y}>S_{m y x}$ then $\mathbf{X}$ depends more on $\mathbf{Y}$ than vice versa, and $\mathbf{Y}$ is considered as 


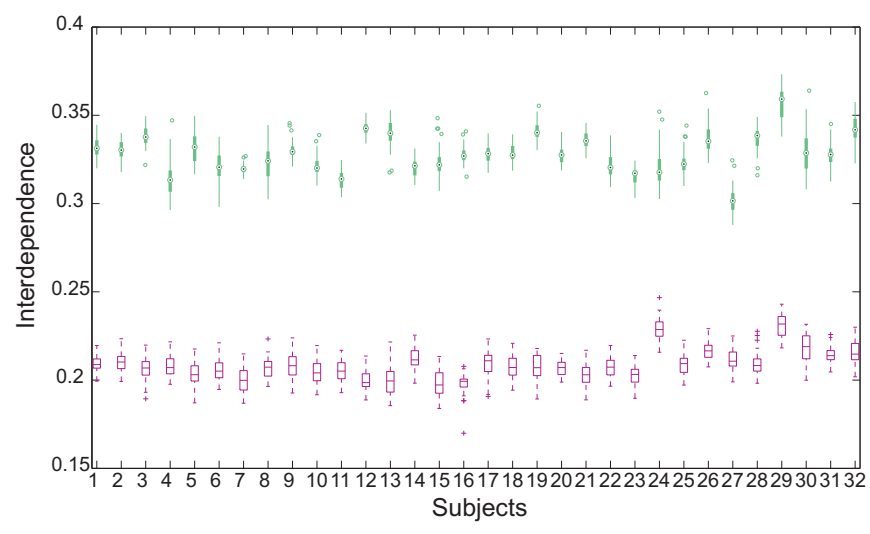

Fig. 4. $S_{m x y}$ and $S_{m y x}$ for each subject. The filled boxes correspond to $S_{m x y}$ and the empty ones to $S_{m y x}$.

more active than $\mathbf{X}$ [15]. In our case, $\mathbf{X}$ represents the EEG and $\mathbf{Y}$ the respiration. Since EEG is generally considered to have a high dimensional attractor, its embedding dimension although estimated using the FNN algorithm, might not be the optimal one. In this case, EEG might have been not completely unfolded, and this could lead to an overestimation of the interdependencies.

From a neurophysiological point of view, emotional stimuli activate the amygdala, which in turn automatically activate networks that control the appearance of visceral responses (autonomic responses such as respiration, skin conductance and hormonal responses) [21]. Visceral responses send back messages to the brain [21], indicating that body dynamics activate brain dynamics. This theory of bodily feedback during emotional reactions was first supported by James [22] who argued, in a nutshell, that we do not cry because we are sad or run because we are afraid, but we are sad because we cry and afraid because we run. Also, Damasio's "somatic marker hypothesis" theory [10] suggests that feedback from the body plays a crucial role in our emotional experiences and decision making. Although the role of bodily feedback is still not fully elucidated [21], we can speculate that the above results (respiration driving frontal EEG signals) corroborate with the bodily feedback process theory.

Regarding the use of this analysis for medical applications, this study can be extended and applied to patients with disorders exposed to affective stimuli to explore the way their coupling patterns are affected, and to compare the results with the ones obtained from healthy subjects.

\section{CONCLUSIONS}

This study investigates the directed synchronization of frontal EEG and respiration while watching music clips. The results reveal a general unidirectional coupling between frontal EEG and respiration, indicating that frontal EEG depends more on respiration than vice versa. This finding corroborates with other neurophysiological studies, that support the idea that body messages activate brain dynamics. As future work the authors are planning to explore how these synchronization phenomena are affected by a relaxation, baseline period without any affective stimuli, and to compare the coupling patterns for the two conditions (affective stimuli and relaxation period).

\section{REFERENCES}

[1] J. Kelso, Dynamic patterns: The self-organization of brain and behavior. MIT press, 1995.

[2] K. Scherer, "Appraisal considered as a process of multilevel sequential checking," Appraisal processes in emotion: Theory, methods, research, vol. 92, pp. 120-128, 2001.

[3] S. Kreibig, "Autonomic nervous system activity in emotion: A review," Biological psychology, vol. 84, no. 3, pp. 394-421, 2010.

[4] M. Dawson, A. Schell, and D. Filion, "The electrodermal system," Handbook of psychophysiology, vol. 2, pp. 200-223, 2000.

[5] Y. Nagai, H. Critchley, E. Featherstone, M. Trimble, and R. Dolan, "Activity in ventromedial prefrontal cortex covaries with sympathetic skin conductance level: a physiological account of a default mode of brain function," Neuroimage, vol. 22, no. 1, pp. 243-251, 2004.

[6] R. Davidson, "What does the prefrontal cortex do in affect: perspectives on frontal EEG asymmetry research," Biological psychology, vol. 67 , no. 1, pp. 219-234, 2004.

[7] J. Coan and J. Allen, "Frontal EEG asymmetry as a moderator and mediator of emotion," Biological psychology, vol. 67, no. 1, pp. 7-50, 2004.

[8] Y. Lin, C. Wang, T. Jung, T. Wu, S. Jeng, J. Duann, and J. Chen, "EEG-based emotion recognition in music listening," Biomedical Engineering, IEEE Transactions on, vol. 57, no. 7, pp. 1798-1806, 2010.

[9] R. Picard, E. Vyzas, and J. Healey, "Toward machine emotional intelligence: Analysis of affective physiological state," Pattern Analysis and Machine Intelligence, IEEE Transactions on, vol. 23, no. 10, pp. 1175-1191, 2001.

[10] A. Damasio, Descartes' error: Emotion, Reason and the Human Brain. Putnam New York, 1994

[11] H. Critchley, R. Melmed, E. Featherstone, C. Mathias, and R. Dolan, "Volitional control of autonomic arousal: a functional magnetic resonance study," Neuroimage, vol. 16, no. 4, pp. 909-919, 2002.

[12] P. Bob, "Hypnotic abreaction releases chaotic patterns of electrodermal activity during dissociation," Intl. Journal of Clinical and Experimental Hypnosis, vol. 55, no. 4, pp. 435-456, 2007.

[13] W. Pritchard and D. Duke, "Measuring chaos in the brain: a tutorial review of nonlinear dynamical EEG analysis," International Journal of Neuroscience, vol. 67, no. 1-4, pp. 31-80, 1992.

[14] G. Valenza, A. Lanata, and E. P. Scilingo, "Oscillations of heart rate and respiration synchronize during affective visual stimulation." Information Technology in Biomedicine, IEEE Transactions on, vol. 16, no. 4, pp. 683-690, 2012.

[15] J. Arnhold, P. Grassberger, K. Lehnertz, and C. Elger, "A robust method for detecting interdependences: application to intracranially recorded EEG," Physica D: Nonlinear Phenomena, vol. 134, no. 4 pp. 419-430, 1999.

[16] H. Fujisaka and T. Yamada, "Stability theory of synchronized motion in coupled-oscillator systems," Progress of Theoretical Physics, vol. 69 , no. 1 , pp. $32-47,1983$.

[17] H. Abarbanel, R. Brown, J. Sidorowich, and L. Tsimring, "The analysis of observed chaotic data in physical systems," Reviews of modern physics, vol. 65, no. 4, p. 1331, 1993

[18] S. Koelstra, C. Muhl, M. Soleymani, J. Lee, A. Yazdani, T. Ebrahimi, T. Pun, A. Nijholt, and I. Patras, "DEAP: A database for emotion analysis; using physiological signals," Affective Computing, IEEE Transactions on, vol. 3, no. 1, pp. 18-31, 2012.

[19] B. Efron and R. J. Tibshirani, An Introduction to the Bootstrap. LinkNew York: Chapman \& Hall, 1993.

[20] M. Whitlock, "Combining probability from independent tests: the weighted z-method is superior to fisher's approach," Journal of evolutionary biology, vol. 18, no. 5, pp. 1368-1373, 2005.

[21] J. LeDoux, The emotional brain: The mysterious underpinnings of emotional life. Simon \& Schuster, 1998.

[22] W. James, The principles of psychology,. Henry Holt and Co, 1890. 